\title{
Estudo Comparativo entre Levobupivacaína a 0,5\% e Bupivacaína Racêmica a 0,5\% Associadas ao Sufentanil na Anestesia Peridural para Cesariana *
}

\section{Comparative Study of 0.5\% Levobupivacaine and 0.5\% Racemic Bupivacaine Associated to Sufentanil in Epidural Anesthesia for Cesarean Delivery}

\author{
João Batista Santos Garcia, TSA ${ }^{1}$, José R Oliveira ${ }^{2}$, Elismar P A Silva ${ }^{3}$, Marcelo S Privado ${ }^{3}$,
} Américo M Yamashita, TSA 4 , Adriana M Issy ${ }^{5}$

\begin{abstract}
RESUMO
Garcia JBS, Oliveira JR, Silva EPA, Privado MS, Yamashita AM, Issy AM - Estudo Comparativo entre Levobupivacaína a 0,5\% e Bupivacaína Racêmica a 0,5\% Associadas ao Sufentanil na Anestesia Peridural para Cesariana
\end{abstract}

Justificativa e Objetivos - Apesar do uso freqüente de anestésicos locais em procedimentos cirúrgicos e obstétricos, a bupivacaína racêmica é associada à cardiotoxicidade potencialmente fatal. Estudos sugerem que a levobupivacaína apresenta ação anestésica local semelhante à bupivacaína racêmica, com a vantagem de menor toxicidade tanto no sistema nervoso central como cardiovascular. Os trabalhos têm demonstrado melhor qualidade anestésica com uso de bupivacaína racêmica associada à sufentanil, via peridural para cesariana. O presente estudo compara a eficácia da bupivacaína racêmica $0,5 \%$ com levobupivacaína 0,5\%, ambas associadas o sufentanil, via peridural, em parturientes submetidas a cesariana.

Método - Foram investigadas 52 gestantes, submetidas à anestesia peridural para cesariana eletiva. Neste estudo duplamente encoberto, as pacientes foram distribuídas aleatoriamente em dois grupos: Grupo I $(n=26)$ : receberam 27 $\mathrm{ml}$ de levobupivacaína 0,5\% e $30 \mu \mathrm{g}$ de sufentanil, Grupo $/ \mathrm{l}(\mathrm{n}=$ 26) receberam $27 \mathrm{ml}$ de bupivacaína 0,5\% e $30 \mu \mathrm{g}$ de sufentanil. Foram avaliados as características dos bloqueios motor e sensorial, o tempo necessário para solicitação de analgésicos e a incidência de efeitos adversos no período pós-operatório.

Resultados - Os bloqueios motor e sensorial, o tempo para solicitação de analgésicos e os efeitos adversos não diferiram entre os grupos. Entretanto, quando se comparou a duração do bloqueio motor da levobupivacaína com da bupivacaína

\footnotetext{
* Recebido do (Received from) Hospital Universitário da Universidade Federal do Maranhão (UFMA) São Luiz, MA

1. Professor Adjunto de Anestesiologia da UFMA

2. Anestesiologista do Hospital Universitário Materno Infantil da UFMA

3. Anestesiologista colaborador

4. Professor Assistente de Anestesiologia da Universidade Federal de São Paulo - UNIFESP - São Paulo -SP

5. Professora Adjunta de Anestesiologia da Universidade Federal de São Paulo - UNIFESP - São Paulo -SP
}

Apresentado (Submitted) em 04 de dezembro de 2000

Aceito (Accepted) para publicação em 21 de março de 2001

Correspondência para (Mail to):

Dr. João Batista Santos Garcia

Av. dos Holandeses, 213/701 - Ponta da Areia

65085-150 São Luiz, MA

(C) Sociedade Brasileira de Anestesiologia, 2001 racêmica, observou-se duração significantemente prolongada para levobupivacaína $(p<0,05)$.

Conclusões - Apesar da duração do bloqueio motor ser mais prolongado para a levobupivacaína associada ao sufentanil, a eficácia anestésica de ambos os anestésicos locais investigados associados ao sufentanil em cesariana por via peridural, foram iguais.

UNITERMOS - ANALGÉSICOS, Opióides: sufentanil; ANESTÉSICOS, Local: bupivacaína, levobupivacaína; CIRURGIA, Obstétrica: cesariana; TÉCNICAS ANESTÉSICAS, Regional: peridural

\section{SUMMARY}

Garcia JBS, Oliveira JR, Silva EPA, Privado MS, Yamashita AM, Issy AM - Comparative Study of $0.5 \%$ Levobupivacaine and $0.5 \%$ Racemic Bupivacaine Associated to Sufentanil in Epidural Anesthesia for Cesarean Delivery

Background and Objectives - Although the widespread use of local anesthetics in surgery and obstetrics, racemic bupivacaine is associated to potentially fatal cardiotoxicity. Data suggest that levobupivacaine has local anesthetic effects similar to racemic bupivacaine with the advantage of less central nervous system and cardiovascular toxicity. Studies have shown that epidural anesthesia with racemic bupivacaine and sufentanil for cesarean sections results in a better quality of anesthesia. This study aimed at comparing the efficacy of $0.5 \%$ racemic bupivacaine and $0.5 \%$ levobupivacaine, both associated to sufentanil, for epidural anesthesia in parturients undergoing cesarean delivery.

Methods - Participated in this double-blind study 52 obstetric patients submitted to elective cesarean delivery under epidural anesthesia. Patients were randomized to receive $27 \mathrm{ml}$ of $0.5 \%$ levobupivacaine and $30 \mu \mathrm{g}$ sufentanil (Group I $\mathrm{n}=26$ ) or $27 \mathrm{ml}$ of $0.5 \%$ bupivacaine and $30 \mu \mathrm{g}$ sufentanil (Group II $n=26$ ). Characteristics of sensory and motor block, time for analgesics request in the postoperative period and the incidence of side effects were investigated.

Results - Sensory and motor block, time for analgesics request and adverse effects did not differ between groups. However, motor block was significantly longer with levobupivacaine as compared to racemic bupivacaine $(p<0.05)$.

Conclusions - Although a longer motor block duration with $0.5 \%$ epidural levobupivacaine associated to sufentanil, the efficacy of both local anesthetics associated to sufentanil for cesarean delivery was similar.

KEY WORDS - ANALGESICS, Opioids: sufentanil; ANESTHETICS, Local: bupivacaine, levobupivacane; ANESTHETIC TECHNIQUES, Regional: epidural; SURGERY, Obstetric: cesarean section 


\section{INTRODUÇÃO}

$A^{\prime}$ levobupivacaína é um enantiômero puro (S-) da bupivacaína, recentemente introduzida no arsenal terapêutico para bloqueios regionais. Apresenta alto grau de ligação às proteínas plasmáticas, elevada depuração e meia-vida de eliminação menor que seu enantiômero $(R+)$. Os enantiômeros (S-) parecem conferir menor cardiotoxicidade sistêmica, com menor incidência de toxicidade para o sistema nervoso central e cardiovascular, segundo estudos pré- clínicos ${ }^{1-3}$.

Estudos clínicos demonstram que a levobupivacaína por via peridural promove bloqueio sensitivo e motor similar ao da bupivacaína (com menor toxicidade) em pacientes que necessitam de anestesia para procedimentos cirúrgicos ${ }^{4}$. Estas características tornam atraente o uso da bupivacaína levógira em anestesia obstétrica, uma vez que as grávidas, em decorrência das adaptações fisiológicas, apresentam risco aumentado de toxicidade.

A associação de anestésicos locais de longa duração por via peridural, aos opióides lipossolúveis e hidrossolúveis além de melhorar a analgesia per e pós-operatória, possibilita o uso de doses menores de anestésicos locais ${ }^{5-9}$.

O objetivo deste estudo é avaliar a eficácia da levobupivacaína e bupivacaína racêmica $0,5 \%$ associada ao sufentanil ( 30 $\mu \mathrm{g})$ por via peridural em pacientes submetidas a cesariana.

\section{MÉTODO}

Após a aprovação do protocolo pela Comissão de Ética do Hospital e do consentimento formal, foram analisadas 52 pacientes, estado físico ASA I ou II, com idades entre 16 e 39 anos, submetidas a cesariana eletiva, sob anestesia peridural.

O estudo foi duplamente encoberto, com distribuição aleatória, dividido em dois grupos de 26 pacientes. O grupo I recebeu $27 \mathrm{ml}$ (135 mg) de levobupivacaína $0,5 \%$ associada a 3 $\mathrm{ml}$ de sufentanil $(30 \mu \mathrm{g})$. O grupo II recebeu $27 \mathrm{ml}(135 \mathrm{mg})$ de bupivacaína racêmica $0,5 \%$ associada a $3 \mathrm{ml}$ de sufentanil $(30 \mu \mathrm{g})$. A administração dos medicamentos em ambos os grupos foi realizada em regime de dose única. Não foi associado vasoconstrictor em nenhum dos grupos. Os fármacos foram distribuídos pelo laboratório em ampolas numeradas, cuja decodificação foi feita ao final do estudo.

Após a venóclise, seguiu-se expansão volêmica com $500 \mathrm{ml}$ de solução de Ringer com lactato. O bloqueio peridural lombar foi realizado através de punção entre $L_{3}-L_{4}$, usando a técnica da perda de resistência ao ar. Após a injeção, as pacientes foram colocadas em posição supina, com deslocamento manual do útero para o lado esquerdo.

Foi realizada monitorização não invasiva da pressão arterial, da freqüência e do ritmo cardíacos, bem como da saturação periférica de oxigênio. A pressão arterial e a freqüência cardíaca foram analisadas em diversos tempos: antes do bloqueio (T0), 5 (T5), 10 (T10), 15 (T15), 20 (T20), 30 (T30), 45 (T45), 60 (T60), 90 (T90), 120 (T120), 180 (T180) e 240 (T240) minutos após a injeção peridural.
Foram observados parâmetros relacionados à instalação e duração do bloqueio, bem como intercorrências e complicações. Em relação à instalação, foi medido e anotado o tempo de ocorrência de parestesias (informação subjetiva da paciente), a latência sensitiva (através de pinçamento na área sensitiva correspondente ao local da punção), o tempo para atingir o nível máximo de bloqueio sensitivo e o nível metamérico de $T_{6}$, além do tempo para alcançar o maior grau de relaxamento muscular (este avaliado pelo teste de relaxamento da musculatura abdominal - RMA, descrito por Van Zudert) ${ }^{10}$. Foram anotados os níveis máximos de bloqueios sensitivos e graus de relaxamento.

Para avaliar a duração do bloqueio, foram estudados os tempos de ocorrência dos seguintes parâmetros:

- anestesia cirúrgica avaliada pelo teste de pinçamento, na mesma área utilizada para pesquisar a sensibilidade inicial (nível da punção);

- relaxamento muscular, revertido quando da recuperação plena da motricidade;

- regressão de dois metâmeros, avaliado pelo tempo necessário para o bloqueio regredir dois segmentos, em relação ao nível sensitivo mais alto obtido;

- duração da analgesia, quando a paciente manifestava, voluntariamente, a necessidade de analgésicos devido a dor no local da operação.

Esses parâmetros foram analisados a partir da injeção da solução anestésica.

As intercorrências foram registradas durante todo o tempo de duração do estudo, tais como hipotensão arterial (queda em $20 \%$ da pressão arterial sistólica inicial), bradicardia (FC $<50$ bpm), náusea e vômito, prurido, sonolência, tremores e calafrios, dor à injeção do anestésico e à extração fetal. A hipotensão arterial foi tratada com doses fracionadas de $10 \mathrm{mg}$ de efedrina.

Para análise estatística dos resultados foram aplicados: teste $t$ de Student para as variáveis paramétricas estudadas e teste de Mann-Whitney para as variáveis não-paramétricas; Análise de variância por postos de Friedman para comparar em cada grupo a freqüência cardíaca, a pressão arterial sistólica (PAS) e a pressão arterial diastólica (PAD) ao longo do tempo; teste do Qui-quadrado ou teste exato de Fisher para estudar as associações das complicações e intercorrências nos grupos ${ }^{11,12}$. Fixou-se em 0,05 ou $5 \%$ o nível de rejeição da hipótese de nulidade.

\section{RESULTADOS}

Não houve diferença significante entre as médias de peso, altura, índice de massa corpórea e idade das pacientes nos dois grupos (Tabela I). 
Tabela I - Dados Demográficos das Pacientes (Média \pm DP)

\begin{tabular}{lcc}
\hline Parâmetros & $\begin{array}{c}\text { Levobupivacaína } \\
(\mathrm{GI})\end{array}$ & $\begin{array}{c}\text { Racêmica } \\
(\mathrm{GII})\end{array}$ \\
\hline Idade (anos) & $26,26 \pm 5.20$ & $23,80 \pm 6,53$ \\
Altura $(\mathrm{cm})$ & $159,69 \pm 6,95$ & $156,54 \pm 7,91$ \\
IMC $\left(\mathrm{kg} \cdot \mathrm{m}^{2}\right)$ & $25,57 \pm 2.99$ & $24,47 \pm 2,38$ \\
Peso $(\mathrm{kg})$ & $65,29 \pm 9,24$ & $62,38 \pm 9,06$ \\
\hline
\end{tabular}

sem significância estatística, testes $t$ de Student e Mann -Whitney

A variação da média da freqüência cardíaca em relação ao tempo mostrou decréscimo significante a partir de $60 \mathrm{mi}-$ nutos para o Gl e 90 minutos para o GII, mas sem diferença significante entre os grupos (Figura 1). Em relação à pressão arterial sistólica, houve decréscimo significante a partir de 15 minutos para o $\mathrm{Gl}$ e a partir de 20 minutos para o GII, não mostrando significância na comparação intergrupos (Figura 2). A análise da pressão arterial diastólica exibiu decréscimo significante para os dois grupos a partir de 20 minutos, também não significante ao compararmos os dois grupos ( Figura 3).

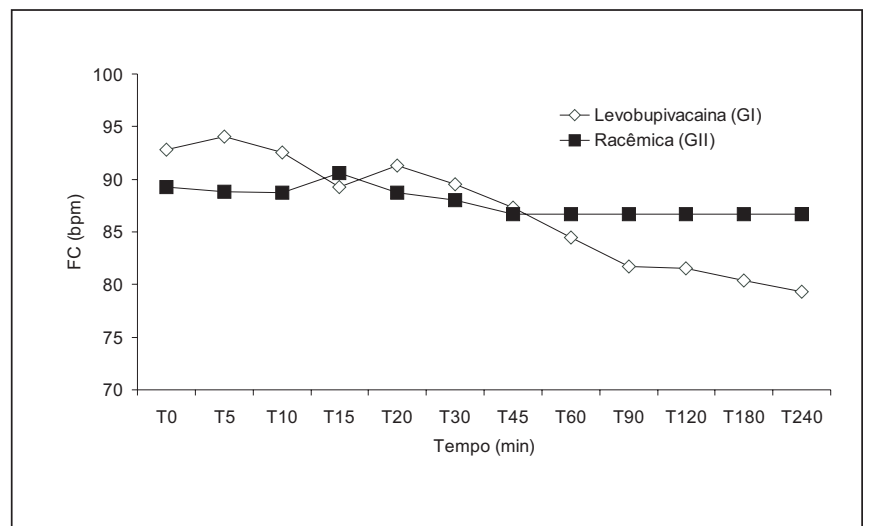

Figura 1 - Valores Médios da Freqüência Cardíaca em Relação ao Tempo

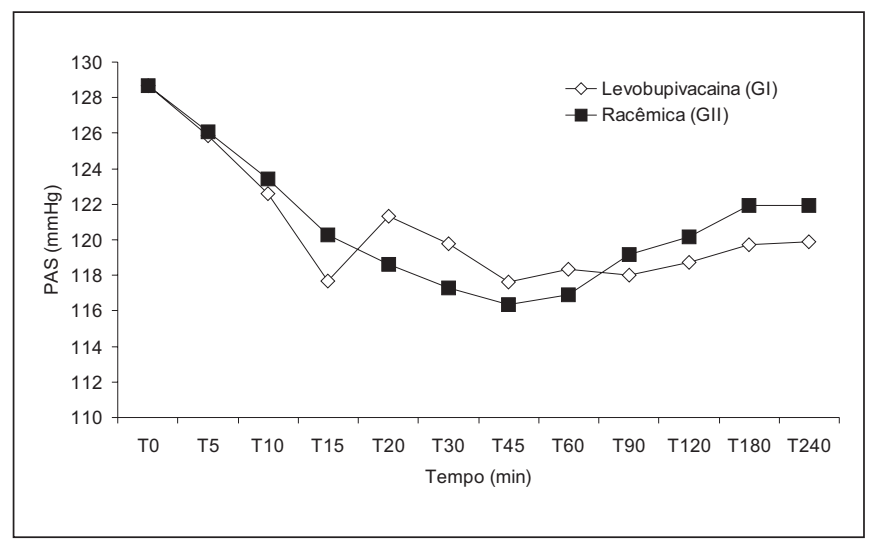

Figura 2 - Valores Médios da Pressão Arterial Sistólica em Relação ao Tempo

Revista Brasileira de Anestesiologia

Vol. 51, № 5, Setembro - Outubro, 2001

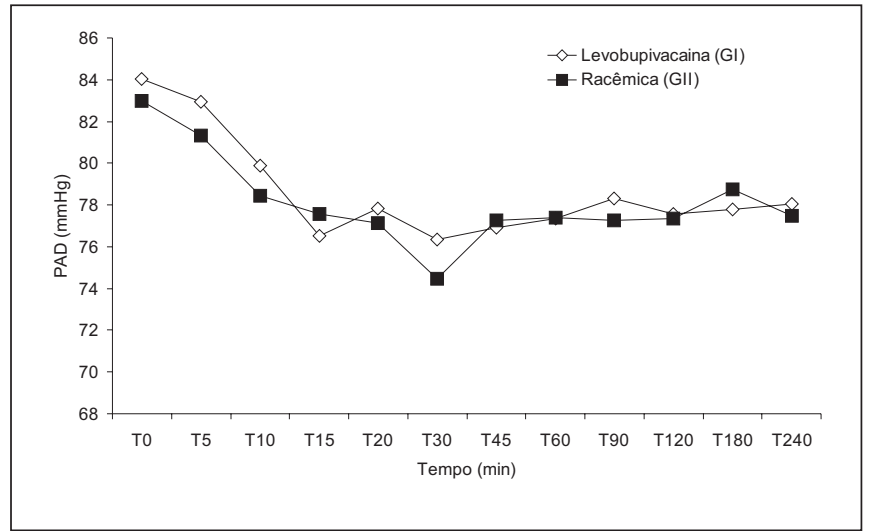

Figura 3 - Valores Médios da Pressão Arterial Sistólica em Relação ao Tempo

As medidas dos tempos de instalação do bloqueio peridural (aparecimento de parestesias, latência sensitiva, tempo para alcançar o nível máximo de bloqueio sensitivo e o maior grau de relaxamento, bem como o tempo para alcançar $\mathrm{T}_{6}$ ) não diferiram de forma significante entre os dois grupos (Tabela II).

Tabela II - Dados Relacionados aos Tempos de Instalação do Bloqueio Peridural (Média \pm DP)

\begin{tabular}{lcc}
\hline Parâmetros & $\begin{array}{c}\text { Levobupivacaína } \\
\text { (GI) }\end{array}$ & $\begin{array}{c}\text { Racêmica } \\
\text { (GII) }\end{array}$ \\
\hline Parestesia (min) & $4,15 \pm 1,56$ & $4,46 \pm 1,30$ \\
Latência sensitiva (min) & $6,88 \pm 2,81$ & $7,85 \pm 2,92$ \\
Nível máximo sensitivo (min) & $18,27 \pm 5, .82$ & $17,88 \pm 4,72$ \\
Nivel máximo relaxamento (min) & $16,92 \pm 4,91$ & $17,12 \pm 5,50$ \\
Nivel $\mathrm{T}_{6}$ (min) & $15,00 \pm 9,68$ & $16,92 \pm 13,71$ \\
\hline
\end{tabular}

sem significância estatística, teste $t$ de Student

Observou-se para ambos os grupos, nível metamérico superior do bloqueio em $\mathrm{T}_{4}$ (moda), com variação de $\mathrm{T}_{8}$ a $\mathrm{T}_{3}$ para

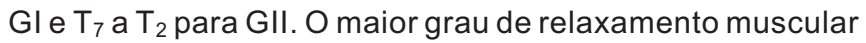
(RMA) foi igual a 3 para os dois grupos.

A duração do relaxamento muscular foi significantemente maior no GI (levobupivacaína)em relação ao GII (racêmica).

A duração da anestesia cirúrgica, do tempo de regressão de dois metâmeros e da necessidade de analgésicos não mostraram diferença significante para os dois grupos (Tabela III).

Tabela III - Dados Relacionados aos Tempos de Duração do Bloqueio Peridural (Média \pm DP)

\begin{tabular}{lcc}
\hline Parâmetros & $\begin{array}{c}\text { Levobupivacaína } \\
\text { (GI) }\end{array}$ & $\begin{array}{c}\text { Racêmica } \\
\text { (GII) }\end{array}$ \\
\hline Anestesia cirúrgica (min) & $200,77 \pm 41,36$ & $201,54 \pm 43,05$ \\
Relaxamento muscular (min) & $303,85 \pm 48,66$ & $272,31 \pm 41,98^{*}$ \\
Tempo de regressão de dois & $147,69 \pm 41,50$ & $148,46 \pm 37,06$ \\
metâmeros (min) & & \\
Necessidade de analgésico (min) & $335,58 \pm 72,61$ & $303,85 \pm 68,54$ \\
\hline
\end{tabular}

* $\mathrm{p}<0,05-\mathrm{GI}>\mathrm{GIII}$, teste $t$ de Student 
Na tabela IV estão apresentadas as intercorrências observadas, que não diferiram significantemente entre os grupos. $O$ prurido foi o efeito adverso mais freqüente (referido por 11 pacientes).

Tabela IV - Dados Relacionados às Intercorrências

\begin{tabular}{lcc}
\hline Parâmetros & $\begin{array}{c}\text { Levobupivacaína } \\
\text { (GI) }\end{array}$ & $\begin{array}{c}\text { Racêmica } \\
\text { (GII) } \\
\text { N e \% }\end{array}$ \\
\hline Hipotensão arterial & $4(15,38 \%)$ & $3(8,33 \%)$ \\
Náusea/vômito & $4(15,38 \%)$ & $4(15.38 \%)$ \\
Prurido & $6(23,08 \%)$ & $5(19,23 \%)$ \\
Sonolência & $5(19,23 \%)$ & $3(08,33 \%)$ \\
Dor à Injeção & $5(19,23 \%)$ & $3(08,33 \%)$ \\
Tremores/calafrios & $2(07,69 \%)$ & $2(07,69 \%)$ \\
Dor à extração fetal & $0(00,00 \%)$ & $3(08,33 \%)$ \\
\hline
\end{tabular}

sem significância estatística , Fisher e Qui-quadrado

\section{DISCUSSÃo}

A ropivacaína e, posteriormente, a levobupivacaína, novos anestésicos locais de longa duração, foram sintetizados com o objetivo de substituir a bupivacaína nas anestesias locorregionais. Ambas apresentam como características menor cardiotoxicidade e neurotoxicidade em relação à bupivacaína ${ }^{13,14}$.

Estudos clínicos têm demonstrado que a levobupivacaína apresenta equipotência com a bupivacaína em termos de eficiência anestésica ${ }^{4,15,16}$.

Seguindo as experiências realizadas com a bupivacaína, os primeiros estudos com ropivacaína e levobupivacaína também associam opióides lipofílicos com o intuito de melhorar a qualidade da anestesia, permitindo reduzir a dose de anestésico local ${ }^{5-9,17,18}$.

Os trabalhos clínicos não têm demonstrado diferença significante quanto ao nível do bloqueio sensitivo, bloqueio motor, relaxamento muscular e qualidade da anestesia entre os grupos que receberam levobupivacaína ou bupivacaína $0,5 \%$ $4,16,19,20$. Os nossos resultados foram semelhantes, entretanto a duração do bloqueio motor foi superior no grupo que recebeu levobupivacaína, sem significado clínico (Tabela III).

Quanto aos tempos de instalação do bloqueio peridural não houve diferença significante entre os grupos na avaliação de latência sensitiva, nível máximo de bloqueio sensitivo, grau máximo de relaxamento muscular e tempo para atingir $T_{6}$ (Tabela II). Nossos resultados foram semelhantes aos observados por outros autores ${ }^{16,17}$.

A associação de opióides aos anestésicos locais nos bloqueios espinhais além de melhorar a qualidade da anestesia, reduz o tempo de instalação do bloqueio ${ }^{5,7,21,22}$.O presente estudo mostrou que a associação de levobupivacaína ou bupivacaína 0,5\% (27 ml) com sufentanil (30 $\mu \mathrm{g})$, proporcionou analgesia adequada para a operação proposta. Apenas três parturientes do grupo da bupivacaína racêmica apresentaram desconforto à extração fetal. O tempo de anal- gesia pós-operatória foi de 355,58 minutos para o grupo da levobupivacaína e 303,35 para o grupo da bupivacaína racêmica, superior quando comparado à administração isolada de bupivacaína racêmica para cesariana.

Em relação à regressão do bloqueio, não houve diferença significante entre os grupos. Resultados semelhantes foram observados por outros autores ${ }^{16}$, em estudo no qual não foi associado opióide lipofílico ao anestésico local.

Quanto às variáveis hemodinâmicas houve redução significante da freqüência cardíaca durante o estudo (T0 - T240), em ambos os grupos, mas não houve diferença entre os grupos (Figura 1). Durante o estudo observou-se significante redução das pressões arteriais sistólica e diastólica nos primeiros 20 minutos, em ambos os grupos. A partir deste momento, observou-se a estabilização destes parâmetros investigados (Figuras 2 e 3). Estas alterações são observações freqüentes durante anestesia regional para cesariana, sem significado clínico .

As intercorrências clínicas não foram relevantes ao ponto de comprometerem a qualidade da anestesia proposta. O prurido foi a intercorrência de maior incidência, conforme a tabela IV, sendo em torno de $50 \%$ menor do que a observada por outros autores ${ }^{17}$ que utilizaram a morfina, além do opióide lipofílico. A hipotensão foi observada em $15,38 \%$ no grupo I e $11,54 \%$ no grupo II, sem diferença entre os grupos, em concordância com os resultados de outros estudos ${ }^{16,17}$.

Concluindo, o uso de levobupivacaína 0,5\% (135 mg) por via peridural associada a sufentanil $(30 \mu \mathrm{g})$ para operações de cesarianas é igualmente eficaz ao uso da associação bupivacaína racêmica $0,5 \%(135 \mathrm{mg})$ e sufentanil $(30 \mu \mathrm{g})$.

\section{Comparative Study of $0.5 \%$ Levobupi- vacaine and $0.5 \%$ Racemic Bupivacaine Associated to Sufentanil in Epidural Anesthesia for Cesarean Delivery}

João Batista Santos Garcia, M.D., José R Oliveira, M.D., Elismar P A Silva M.D., Marcelo S Privado, M.D., Américo M Yamashita, M.D., Adriana M Issy M.D.

\section{INTRODUCTION}

Levobupivacaine is a bupivacaine's pure enantiomer (S-) recently introduced in the therapeutic armamentarium for regional blocks. It is strongly bound to plasma proteins with high clearance rate and shorter half-life as compared to its enantiomer $(\mathrm{R}+)$. Enantiomers (S-) seem to cause less systemic cardiotoxicity, with a lower incidence of central nervous system and cardiovascular toxicity according to pre-clinical studies $^{1-3}$.

Clinical studies have shown that epidural levobupivacaine promotes sensory and motor block similar to bupivacaine (with lower toxicity) in patients needing surgical anesthesia ${ }^{4}$.

Revista Brasileira de Anestesiologia Vol. 51, N 5, Setembro - Outubro, 2001 
These characteristics make levogyrous bupivacaine attractive for obstetric anesthesia since pregnant women have a high toxicity risk due to physiological adaptations.

The association of long-lasting epidural anesthetics and liposoluble or hydrosoluble opioids, in addition to improving peri and postoperative analgesia, allows for the use of lower local anesthetic doses ${ }^{5-9}$.

This study aimed at evaluating the efficacy of epidural $0.5 \%$ racemic levobupivacaine and bupivacaine, both associated to sufentanil $(30 \mu \mathrm{g})$ in patients submitted to cesarean delivery.

\section{METHODS}

After the Hospital's Ethics Committee approval and their formal consent, participated in this double-blind study 52 patients, physical status ASA I or II, aged 16 to 39 years, submitted to elective cesarean delivery under epidural anesthesia. Patients were randomly distributed in two groups of 26 patients each. Group I received $27 \mathrm{ml}$ (135 mg) of $0.5 \%$ levobupivacaine associated to $3 \mathrm{ml}$ sufentanil $(30 \mu \mathrm{g})$ and Group II received $27 \mathrm{ml}(135 \mathrm{mg}$ ) of $0.5 \%$ racemic bupivacaine associated to $3 \mathrm{ml}$ sufentanil $(30 \mu \mathrm{g})$, both in a single dose. No vasoconstrictor was associated for both groups. Drugs were distributed by the laboratory in numbered vials which were decoded at the end of the study.

Venoclysis was followed by volume expansion with $500 \mathrm{ml}$ lactated Ringer's. Lumbar epidural block was performed at $\mathrm{L}_{3}-\mathrm{L}_{4}$ interspace using the loss of resistance to air technique. After injection, patients were placed in the supine position with manual uterus displacement to the left.

Monitoring consisted of non-invasive blood pressure, heart rate and rhythm and oxygen peripheral saturation. Blood pressure and heart rate were evaluated in different moments: before blockade (T0), 5 (T5), 10 (T10), 15 (T15), 20 (T20), 30 (T30), 45 (T45), 60 (T60), 90 (T90), 120 (T120), 180 (T180) and 240 (T240) minutes after epidural injection.

Parameters related to blockade installation, in addition to intercurrences and complications were observed. For installation, paresthesias (patients subjective information), sensory onset (by clamping the sensitive area corresponding to puncture site), time for maximum sensory block and metameric level of $T_{6}$, in addition to time for maximum muscular relaxation (confirmed by abdominal muscles relaxation test - AMR, described by Van Zudert) ${ }^{10}$ were measured and recorded.

To evaluate blockade duration, times for the following parameters were studied:

- surgical anesthesia evaluated by the clamping test, in the same area used to check initial sensitivity (puncture level);

- muscular relaxation, reverted with total motility recovery;

- regression of two metamers, evaluated by the time needed for the blockade to regress two segments, as compared to the highest blockade obtained;
- analgesia duration, when the patient would voluntarily ask for analgesics due to pain on surgery site.

Those parameters were analyzed as from anesthetic solution injection.

Intercurrences, such as hypotension (20\% below initial systolic blood pressure), bradycardia ( $\mathrm{HR}<50 \mathrm{bpm})$, nausea and vomiting, pruritus, somnolence, shivering, pain on anesthetic injection site and at fetal extraction, were recorded during the whole study. Hypotension was treated with fractionated doses of $10 \mathrm{mg}$ ephedrine.

For statistical analysis of results Student's $t$ test for parametric variables and Mann-Whitney test for non-parametric variables were applied. Friedman's analysis of variance by posts was used to compare heart rate, systolic blood pressure (SBP) and diastolic blood pressure (DBP) within each group along time. Chi-square or Fisher exact test were applied to study complications and intercurrence associations within groups ${ }^{11,12}$. Null hypothesis rejection level was established as 0.05 or $5 \%$.

\section{RESULTS}

There were no significant differences in mean weight, height, body mass index and age in both groups (Table I).

Table I - Demographics Data (Mean \pm SD)

\begin{tabular}{lcc}
\hline Parameters & $\begin{array}{c}\text { Levobupivacaine } \\
(\mathrm{GI})\end{array}$ & $\begin{array}{c}\text { Racemic } \\
(\mathrm{GII})\end{array}$ \\
\hline Age (years) & $26.26 \pm 5.20$ & $23.80 \pm 6.53$ \\
Height $(\mathrm{cm})$ & $159.69 \pm 6.95$ & $156.54 \pm 7.91$ \\
BMl $\left(\mathrm{kg} . \mathrm{m}^{2}\right)$ & $25.57 \pm 2.99$ & $24.47 \pm 2.38$ \\
Weight $(\mathrm{kg})$ & $65.29 \pm 9.24$ & $62.38 \pm 9.06$ \\
\hline
\end{tabular}

Without statistical significance, Student's $t$ and Mann-Whitney tests

Mean heart rate variation along time has shown a significant decrease as from 60 minutes for $\mathrm{GI}$ and 90 minutes for $\mathrm{GII}$, without significant differences between groups (Figure 1).

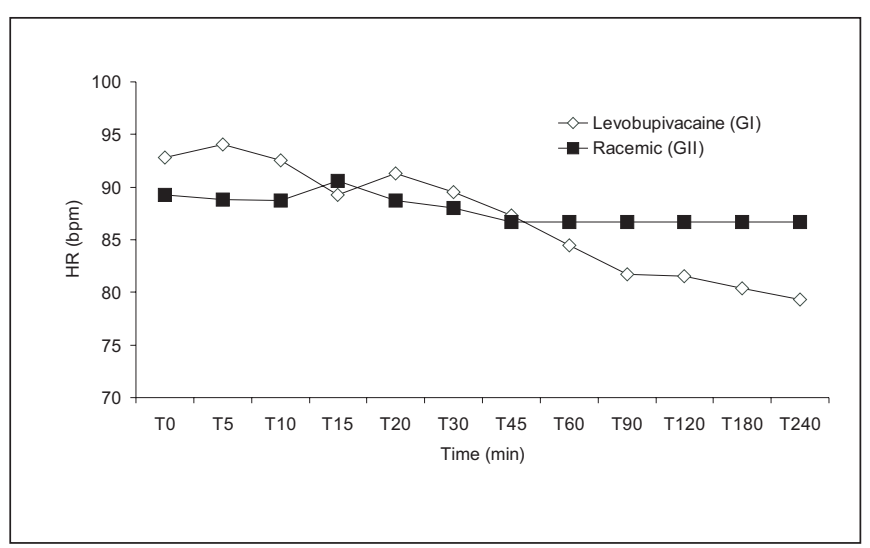

Figure 1 - Mean Heart Rate Values Compared to Time 
There has been a significant systolic blood pressure decrease as from 15 minutes for $\mathrm{GI}$ and 20 minutes for GII, without significance when comparing both groups (Figure 2). Diastolic blood pressure significantly decreased in both groups as from 20 minutes, also without significance when comparing both groups (Figure 3 ).

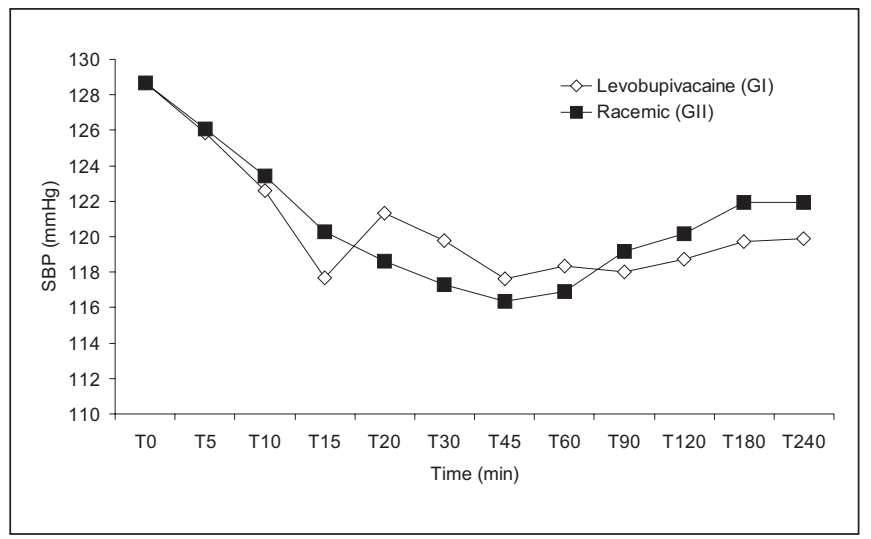

Figure 2 - Mean Systolic Blood Pressure Values Compared to Time

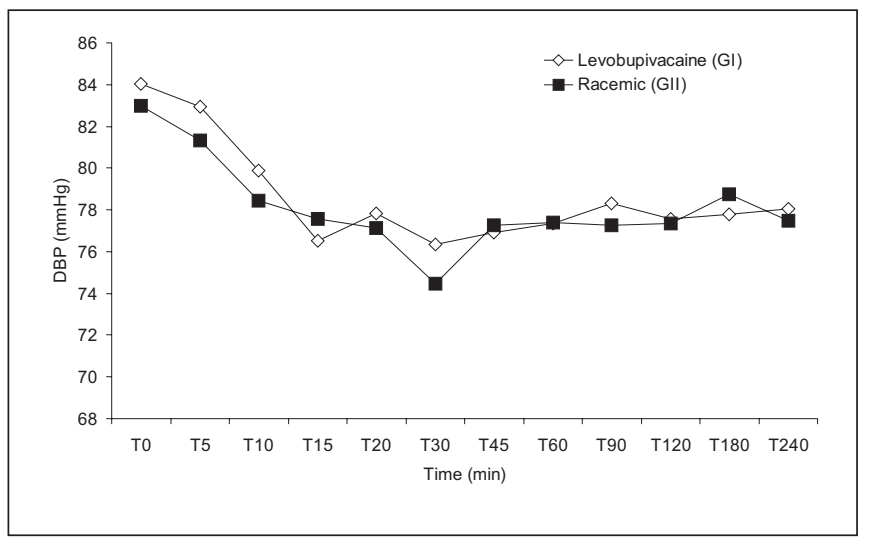

Figure 3 - Mean Diastolic Blood Pressure Values Compared to Time

Epidural blockade onset measurements (paresthesias, sensory onset, time for maximum sensory block and relaxation, as well as time for reaching $T_{6}$ ) were not significantly different between groups (Table II).

Table II - Epidural Blockade Installation Time (Mean \pm SD)

\begin{tabular}{lcc}
\hline Parameters & $\begin{array}{c}\text { Levobupivacaine } \\
(\mathrm{Gl})\end{array}$ & $\begin{array}{c}\text { Racemic } \\
(\mathrm{GII})\end{array}$ \\
\hline Paresthesia (min) & $4.15 \pm 1.56$ & $4.46 \pm 1.30$ \\
Sensory onset (min) & $6.88 \pm 2.81$ & $7.85 \pm 2.92$ \\
Maximum sensory (min) & $18.27 \pm 5 . .82$ & $17.88 \pm 4.72$ \\
Maximum relaxation (min) & $16.92 \pm 4.91$ & $17.12 \pm 5.50$ \\
$\mathrm{~T}_{6}$ (min) & $15.00 \pm 9.68$ & $16.92 \pm 13.71$ \\
\hline
\end{tabular}

Without statistical significance Student's $t$ test
Ametameric level above blockade in $\mathrm{T}_{4}$ (mode) was observed in both groups, with variation of $T_{8}$ to $T_{3}$ in $G I$ and of $T_{7}$ to $T_{2}$ in GII. Highest muscular relaxation level (MRL) was 3 for both groups. The muscular relaxation duration was significantly higher in GI (levobupivacaine) as compared to GII (racemic). Anesthesia duration, time for two-metamer regression and for analgesic request were not significantly different between groups (Table III).

Table III - Epidural Blockade Duration (Mean \pm SD)

\begin{tabular}{lcc}
\hline Parameters & $\begin{array}{c}\text { Levobupivacaine } \\
(\mathrm{GI})\end{array}$ & $\begin{array}{c}\text { Racemic } \\
(\mathrm{GII})\end{array}$ \\
\hline Surgical anesthesia $(\mathrm{min})$ & $200.77 \pm 41.36$ & $201.54 \pm 43.05$ \\
Muscular relaxation $(\mathrm{min})$ & $303.85 \pm 48.66$ & $272.31 \pm 41.98^{*}$ \\
$\begin{array}{l}\text { Two metamers regression } \\
\text { time (min) }\end{array}$ & $147.69 \pm 41.50$ & $148.46 \pm 37.06$ \\
Analgesics request (min) & $335.58 \pm 72.61$ & $303.85 \pm 68.54$ \\
\hline
\end{tabular}

*: $p<0.05-$ GI $>$ GIII, Student's $t$ test

Intercurrences, which were not significantly different between groups are shown in Table IV. Pruritus was the most frequent adverse effect (referred by 11 patients).

Table IV - Intercurrences

\begin{tabular}{lcc}
\hline Parameters & $\begin{array}{c}\text { Levobupivacaine } \\
(\mathrm{GI}) \\
\mathrm{n} \text { and } \%\end{array}$ & $\begin{array}{c}\text { Racemic } \\
(\mathrm{GII}) \\
\mathrm{n} \text { and } \%\end{array}$ \\
\hline Hypotension & $4(15.38 \%)$ & $3(8.33 \%)$ \\
Nausea/Vomiting & $4(15.38 \%)$ & $4(15.38 \%)$ \\
Pruritus & $6(23.08 \%)$ & $5(19.23 \%)$ \\
Somnolence & $5(19.23 \%)$ & $3(08.33 \%)$ \\
Pain on injection & $5(19.23 \%)$ & $3(08.33 \%)$ \\
Shivering & $2(07.69 \%)$ & $2(07.69 \%)$ \\
Pain on fetal extraction & $0(00.00 \%)$ & $3(08.33 \%)$ \\
\hline
\end{tabular}

Without statistical significance, Fisher and Chi-square

\section{DISCUSSION}

Ropivacaine, and subsequently levobupivacaine, new long-lasting local anesthetics, were synthesized to replace bupivacaine in local/regional blocks. Both have less cardiotoxicity and neurotoxicity as compared to bupivacaine ${ }^{13,14}$.

Clinical studies have shown that levobupivacaine is equipotent to bupivacaine in terms of anesthetic efficacy $4,15,16$. In line with bupivacaine experiments, early studies with ropivacaine and levobupivacaine have also associated lipophylic opioids aiming at improving anesthesia and decreasing the dose of local anesthetics ${ }^{5-9,17,18}$. Clinical trials have not shown significant differences in sensory block, motor block, muscle relaxation and anesthesia quality between groups receiving $0.5 \%$ levobupivacaine or bupivacaine ${ }^{4,16,19,20}$. Our results were similar, but the motor block duration was higher in the levobupivacaine group, without clinical significance (Table III).

Revista Brasileira de Anestesiologia Vol. 51, № 5, Setembro - Outubro, 2001 
As to time to epidural block installation, there have been no significant differences between groups in sensory onset, maximum sensory block, maximum muscular relaxation and time to reach $\mathrm{T}_{6}$ (Table II). Our results were similar to other authors' 16,17 .

The association of opioids to local anesthetics in spinal blocks decreases blockade onset time and improves anesthesia quality $5,7,21,22$. Our study has shown that the association of $0.5 \%$ levobupivacaine or bupivacaine $(27 \mathrm{ml})$ to sufentanil $(30 \mu \mathrm{g})$, has provided an adequate analgesia for the proposed surgery. Only three racemic group patients referred discomfort at fetal extraction. Postoperative analgesia was 355.58 minutes for the levobupivacaine group and 303.35 for the racemic bupivacaine group, and longer as compared to the administration of racemic bupivacaine alone for cesarean delivery.

There have been no significant differences in blockade regression between groups. Similar results were obtained by other authors ${ }^{16}$ in a study where no lipophylic opioid was associated to local anesthetics.

Both groups presented a significant heart rate decrease during the study (T0 -T240), but without differences between groups (Figure 1). A significant systolic and diastolic blood pressure decrease was observed during the first 20 minutes in both groups. As from this moment there has been a stabilization of such parameters (Figures 2 and 3 ). These are common events during regional anesthesia for cesarean delivery and have no clinical significance.

Clinical intercurrences were irrelevant to the point that they did not compromise anesthesia quality. Pruritus was the most frequent complaint, as shown in table IV and was approximately $50 \%$ lower than what has been observed by other authors ${ }^{17}$ using morphine in addition to lipophylic opioids. Hypotension was present in $15.38 \%$ of Group I and $11.54 \%$ of Group II patients, without differences between groups and in line with other studies ${ }^{16,17}$.

We concluded that epidural $0.5 \%$ levobupivacaine $(135 \mathrm{mg}$ ) associated to sufentanil $(30 \mu \mathrm{g})$ for cesarean delivery is as effective as the association of $0.5 \%$ racemic bupivacaine (135 $\mathrm{mg})$ and sufentanil $(30 \mu \mathrm{g})$.

\section{REFERÊNCIAS - REFERENCES}

01. Denson DD, Behbehani MM, Gregg RV - Enantiometer-specific effects of an intravenously administered arrythmogenic dose of bupivacaine on neurons of the nucleus tractus solitarius and the cardivascular system in the anesthetized rat. Reg Anesth, 1992;17:311-316.

02. Nancarrow C, Rutten AJ, Runciman WB et al - Myocardial and cerebral drug concentrations and the mechanisms of death after fatal intravenous doses of lidocaine, bupivacaine, and ropivacaine in the sheep. Anesth Analg, 1989;69:276-283.

03. Feldman HS, Arthur GR, Covino BG - Comparative systemic toxicity of convulsant and supraconvulsant doses of intravenous ropivacaine, bupivacaine, and lidocaine in the conscious dog. Anesth Analg, 1989;69:794-801.

04. Cox CR, Faccenda KA, Gilhooly C et al - Extradural $\mathrm{S}(-)$-bupivacaine: comparison with racemic $\mathrm{RS}$-bupivacaine. $\mathrm{Br}$ J Anaesth, 1989;80:289-293.
05. Naulty JS, Datta S, Ostheimer GW et al - Epidural fentanyl for postcesarean delivery pain management. Anesthesiology, 1985;63:694-698.

06. Jones G, Paul DL, Elton RA et al - Comparison of bupivacaine and bupivacaine with fentanyl in continuous extradural analgesia during labour. Br J Anaesth, 1989;63:254-259.

07. McMorland GH, Douglas MJ, Kim JHK et al - Epidural sufentanil for post-Cesaean section analgesia: lack of benefit of epinephrine. Can J Anaesth, 1990;37:432-437.

08. De Leon-Casasola AO, Lema MJ - Postoperative epidural opioid analgesia: what are the choices? Anesth Analg, 1996;83: 867-875.

09. Crews JC, Hord AH, Denson DD et al - A comparison of the analgesic efficacy of $0.25 \%$ levobupivacaine combined with $0.005 \%$ morphine, $0.25 \%$ levobupivacaine alone, or $0.005 \%$ morphine alone for the management of postoperative pain in patients undergoing major abdominal surgery. Anesth Analg, 1999;89: 1504-1509.

10. Van Zudert A, Vaes L, Van der AAP et al - Motor blockade during epidural anesthesia. Anesth Analg, 1986;65:333-336.

11. Sokal RR, Rohlf FJ - Biometry. San Francisco, WH Freeman, $1969 ; 776$

12. Siegel S, Castellan Jr NJ - Nonparametric Statistics. $2^{\text {nd }}$, New York, McGraw-Hill, 1988; 399.

13. Bardsley H, Gristwood R, Baker H et al - A comparison of the cardiovascular effects of levobupivacaine following intravenous administration to healthy volunteers. $\mathrm{Br} \mathrm{J}$ Clin Pharmacol, 1998;46:245-249.

14. Thomas JM, Stephan AS - Recent advances in the pharmacokinetics of local anaesthetics. Long-acting amide enantiomers and continuous infusions. Clin Pharmacokinet, 1999;36:67-83

15. Cox CR, Checketts MR, Mackenzie N - Comparison of $\mathrm{S}(-)$-bupivacaine with racemic (RS)-bupivacaine in supraclavicular brachial plexus block. Br J Anaesth, 1998;80: 594-598.

16. Bader AM, Tsen LC, Camman WR et al - Clinical effects and maternal and fetal plasma concentrations of $0.5 \%$ epidural levobupivacaine versus bupivacaine for cesarean delivery. Anesthesiology, 1999;90:1596-1601.

17. Delfino J, Vale NB, Magalhães Filho E - $0.45 \%$ ropivacaine and levobupivacaine associated to opioids in epidural anesthesia for cesarean section: a comparative study. Rev Bras Anestesiol, 1999;49:244-248.

18. McClellan KJ, Spencer CM - Levobupivacaine. Drugs 1998;56:355-362.

19. Van Steenberge A, Debroux HC, Noorduin H - Extradural bupivacaine with sufentanil for vaginal delivery. $\mathrm{Br} \mathrm{J}$ Anaesth, 1987;59:1518-1522.

20. Johnson C, Oriol N - Comparison of onset time between bupivacaine $0.5 \%$ and 2 -chloroprocaine 35 with and without fentanyl 75mcg. Anesth Analg, 1990;70:S179.

\section{RESUMEN}

Garcia JBS, Oliveira JR, Silva EPA, Privado MS, Yamashita AM, Issy AM - Estudio Comparativo entre Levobupivacaína a $0,5 \%$ y Bupivacaína Racémica a $0,5 \%$ Asociadas al Sufentanil en la Anestesia Peridural para Cesárea

Justificativa y Objetivos - A pesar del uso frecuente de anestésicos locales en procedimientos quirúrgicos $y$ obstétricos, la bupivacaína racémica es asociada a la cardiotoxicidad potencialmente fatal. Estudios sugieren que la levobupivacaína presenta acción anestésica local semejante a 
la bupivacaína racémica, con la ventaja de menor toxicidad tanto en el sistema nervioso central como cardiovascular. Los trabajos han demostrado mejor calidad anestésica con el uso de bupivacaína racémica asociada al sufentanil, vía peridural para cesárea. El presente estudio compara la eficacia de la bupivacaína racémica $0,5 \%$ con levobupivacaína $0,5 \%$, ambas asociadas al sufentanil, vía peridural, en parturientas sometidas a cesárea.

Método - Fueron investigadas 52 mujeres embarazadas, sometidas a anestesia peridural para cesárea electiva. En este estudio duplamente encubierto, las pacientes fueron distribuidas aleatoriamente en dos grupos: Grupo I $(n=26)$. recibieron $27 \mathrm{ml}$ de levobupivacaína $0,5 \%$ y $30 \mu \mathrm{g}$ de sufentanil, Grupo II $(n=26)$ recibieran $27 \mathrm{ml}$ de bupivacaína 0,5\% y $30 \mu \mathrm{g}$ de sufentanil. Fueron evaluadas las características de los bloqueos motor y sensorial, el tiempo necesario para solicitación de analgésicos y la incidencia de efectos adversos en el período pós-operatorio.

Resultados - Los bloqueos motor y sensorial, el tiempo para solicitación de analgésicos y los efectos adversos no divergieron entre los grupos. Entretanto, cuando se comparó la duración del bloqueo motor de la levobupivacaína con el de la bupivacaína racémica, se observó duración significantemente prolongada para levobupivacaína $(p<0,05)$.

Conclusiones - A pesar de la duración del bloqueo motor ser más prolongado para la levobupivacaína asociada al sufentanil, la eficacia anestésica de ambos anestésicos locales investigados, asociados al sufentanil en cesárea por vía peridural, fueron iguales. 